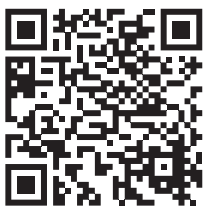

\footnotetext{
Palabras clave: Simulación, simulación quirúrgica, cadáver humano, fantomas.

Keywords: Simulation, surgical simulation, human corpse, phantoms.
}

* Prof. Agregado de Clínica Quirúrgica 3. Prof. Agregado del Departamento de Anatomía.

¥ Asistente de Clínica Quirúrgica 3.

$\S$ Residente de cirugía general en la Clínica Quirúrgica 3. Asistente del Departamento de Anatomía.

॥ Prof. Director de

Clínica Quirúrgica 3.

Facultad de Medicina de la Universidad de la República. Uruguay.

Recibido: 01/03/2020

Aceptado: 18/07/2020

doi: $10.35366 / 95230$

\title{
Entrenamiento quirúrgico en herniorrafias y hernioplastias inguinales utilizando cadáveres humanos como modelo simulador
}

\author{
Surgical training in herniorrhaphy and inguinal hernioplasties \\ using human corpses as a simulator model
}

Gustavo Armand Ugon-Bigi,* Cecilia Chambón-Moleda, ${ }^{\ddagger}$ Santiago Pose-Veirano, ${ }^{\S}$ Daniel González-González"

\section{RESUMEN}

Introducción: En el laboratorio de destrezas quirúrgicas del Hospital Maciel, los residentes de cirugía general se entrenan utilizando simuladores de diferente complejidad y fidelidad. Con ello adquieren y desarrollan diferentes destrezas en un ambiente seguro y controlado. Esto aumenta la confianza y seguridad del residente en la realización de maniobras quirúrgicas y repercute positivamente en la seguridad del paciente. Las hernias inguinales tienen alta incidencia y su tratamiento, exclusivamente quirúrgico, se sustenta en un sólido conocimiento anatomoquirúrgico. Objetivo: Comunicar nuestra experiencia en la utilización de cadáveres humanos para el entrenamiento de herniorrafias y hernioplastias inguinales. Material y métodos: 24 regiones inguinales de cadáveres adultos formolados (ambos sexos), realizamos herniorrafias y hernioplastias por abordaje laparotómico y laparoscópico. Resultados: La rigidez del material cadavérico no fue un impedimento para la realización del ejercicio quirúrgico, pero requirió realizar abordajes más amplios. Los residentes evaluaron positivamente el entrenamiento quirúrgico, pudieron familiarizarse con la anatomía quirúrgica de la región, realizaron, repitieron y sistematizaron cada técnica quirúrgica en similares condiciones espaciales y físicas que en el paciente. Conclusiones: El cadáver humano es un excelente fantoma para el entrenamiento en herniorrafias y hernioplastias. La rigidez del material no fue una limitante para el ejercicio de simulación propuesto.

\section{ABSTRACT}

Introduction: In the surgical skills laboratory of the Maciel Hospital, general surgery residents are trained using simulators of different complexity and fidelity. They acquire and develop different skills in a safe and controlled environment. This increase the confidence and security of the resident in performing surgical maneuvers and has positive impact on patient safety. Inguinal hernias have a high incidence and their treatment, exclusively surgical, is based on a solid anatomo-surgical knowledge. Objective: To communicate our experience in the use of human corpses for the training of inguinal herniorrhaphy and hernioplasties. Material and methods: 24 inguinal regions of adult corpses fixed in formalin (both sexes), we performed herniorrhaphy and hernioplasty by laparotomic and laparoscopic approach. Results: The rigidity of the cadaveric material was not an impediment to the performance of the surgical exercise but required broader approaches. The residents positively assessed the surgical training, were able to familiarize themselves with the surgical anatomy of the region, performed, repeated and systematized each surgical technique in similar spatial and physical conditions as in the patient. Conclusions: The human corpse is an excellent phantom for training in herniorrhaphy and hernioplasty. The rigidity of the material wasn't a limitation for the proposed simulation exercise.

\section{INTRODUCCIÓN}

$\mathrm{L}$ a utilización de simulación en el entrena- miento quirúrgico aparece a lo largo de la historia de la cirugía, estando fechados los primeros registros de utilización de un modelo simulador en el siglo III a.C. con el cirujano hindú Sushruta. No obstante ese y otros ejemplos, la enseñanza de la cirugía en los dos últimos milenios estuvo centrada en el sistema de maestro y aprendices, basado en la enseñanza y la práctica directa sobre el paciente. ${ }^{1,2}$

Desde fines del siglo XX, y como consecuencia de la aparición de nuevas tecnologías, entre ellas la videocirugía y las suturas mecánicas, pero también consecuencia del cambio experimentado 
tanto por los pacientes como por las autoridades de salud en cuanto a calidad y seguridad de la atención sanitaria, es que se establecen y desarroIlan los diferentes programas de simulación como parte del entrenamiento quirúrgico. ${ }^{1-5}$

Hoy en día, la simulación como parte del entrenamiento quirúrgico está incorporada a los programas de formación del residente y a aquéllos de actualización de los cirujanos. Es un complemento esencial y un paso previo ineludible del sistema tutorial establecido en el siglo XIX por William Halsted. Sistema basado en la adquisición progresiva de responsabilidades por parte del cirujano en formación, tutorizado por un cirujano de mayor experiencia, pero entrenando directamente sobre el paciente. ${ }^{2,3,5,6}$

El laboratorio de simulación quirúrgica permite el desarrollo de habilidades psicomotoras en un ambiente seguro y controlado. El residente, además de adquirir destrezas quirúrgicas, se familiariza con los diferentes instrumentos e insumos que utilizará en el quirófano. La estandarización y la repetición de los diferentes ejercicios, enfocados en el residente y su aprendizaje, permiten alcanzar altos niveles de entrenamiento, lo que mejora la seguridad de los pacientes ya que disminuye errores $y$, por tanto, potenciales lesiones. . $^{1,4,5,7-9}$

Existen múltiples dispositivos para el entrenamiento quirúrgico, pueden ser simples o muy complejos, tener mayor o menor fidelidad, etcétera. Los cadáveres humanos, frescos o sometidos a algún proceso de fijación y conservación, constituyen un insumo de altísima calidad para el entrenamiento quirúrgico, tanto para procedimientos laparotómicos como laparoscópicos. Con la ventaja adicional de permitir la simulación del procedimiento quirúrgico completo. 1,4,5,7-9

El cadáver humano, como modelo de simulación, tiene muchas ventajas, ofrece una igual realidad espacial (en cuanto a dimensiones) y una similar realidad física en comparación con el paciente. Permite reconocer y familiarizarse con las estructuras anatómicas propias de la región a intervenir, así como reproducir paso a paso una técnica quirúrgica. También permite adquirir destrezas en el manejo del instrumental quirúrgico en una realidad muy similar a la "in vivo". Todo ello en un ambiente seguro donde no habrá complicaciones. ${ }^{4,8,9}$

El cadáver humano, como modelo de simulación, tiene algunas desventajas, como la no circulación de fluidos y la rigidez (en el caso de material formolado). Rigidez que puede dificultar la realización de alguna maniobra y/o el posicionamiento de la pieza anatómica (para recrear la posición quirúrgica), pero que pueden compensarse con abordajes más amplios, evisceración parcial de cavidades, etcétera, exige una especial adecuación del material cadavérico a la práctica a realizar. ${ }^{5,8,9}$

En el Hospital Maciel contamos con un laboratorio de entrenamiento quirúrgico, equipado con simuladores de diferente complejidad y fidelidad. Laboratorio que puede ser utilizado tanto por residentes como por cirujanos.

Los residentes de cirugía general realizan cursos obligatorios de entrenamiento quirúrgico, tanto en habilidades laparoscópicas como laparotómicas.

Las hernias inguinales tienen alta incidencia y su tratamiento, exclusivamente quirúrgico, se sustenta en un sólido conocimiento anatomoquirúrgico. ${ }^{10,11}$

En la Clínica Quirúrgica Núm. 3 las herniorrafias y las hernioplastias mediante abordaje laparotómico son realizadas por residentes de cirugía general en su primer año de formación, mientras que las hernioplastias mediante abordaje laparoscópico son realizadas por residentes a partir de su segundo año. Las técnicas más utilizadas son las hernioplastias de Lichtenstein (tanto para hernia inguinal como para hernia crural) y la transabdominal preperitoneal (TAAP). En el contexto de la cirugía de urgencia, cuando no es segura la realización de una hernioplastia por el riesgo potencial de infección de la malla, las técnicas más utilizadas son la herniorrafia de Bassini o de Shouldice (para hernia inguinal) y la de Ellis o Devlin (para hernia crural). Todas estas técnicas quirúrgicas son realizadas en el laboratorio de entrenamiento quirúrgico utilizando material cadavérico humano. ${ }^{11-14}$

Objetivo: comunicar nuestra experiencia en la utilización de material cadavérico humano para el entrenamiento quirúrgico en diferentes técnicas de herniorrafia y hernioplastia inguinal y crural.

\section{MATERIAL Y MÉTODOS}

Utilizamos 24 regiones inguinales de cadáveres adultos formolados, de ambos sexos, provenientes del Dpto. de Anatomía de la Facultad de Medicina, regiones que podían estar en una pelvis entera o una hemipélvis, pero todas conservaban sin disecar las regiones inguinoabdominal e inguinocrural y el tercio inferior de la pared abdominal.

Utilizamos un equipo de laparoscopia (Olympus ${ }^{\circledR}$ ) y dos pelvitrainers (Training Competence ${ }^{\circledR}$ ), 

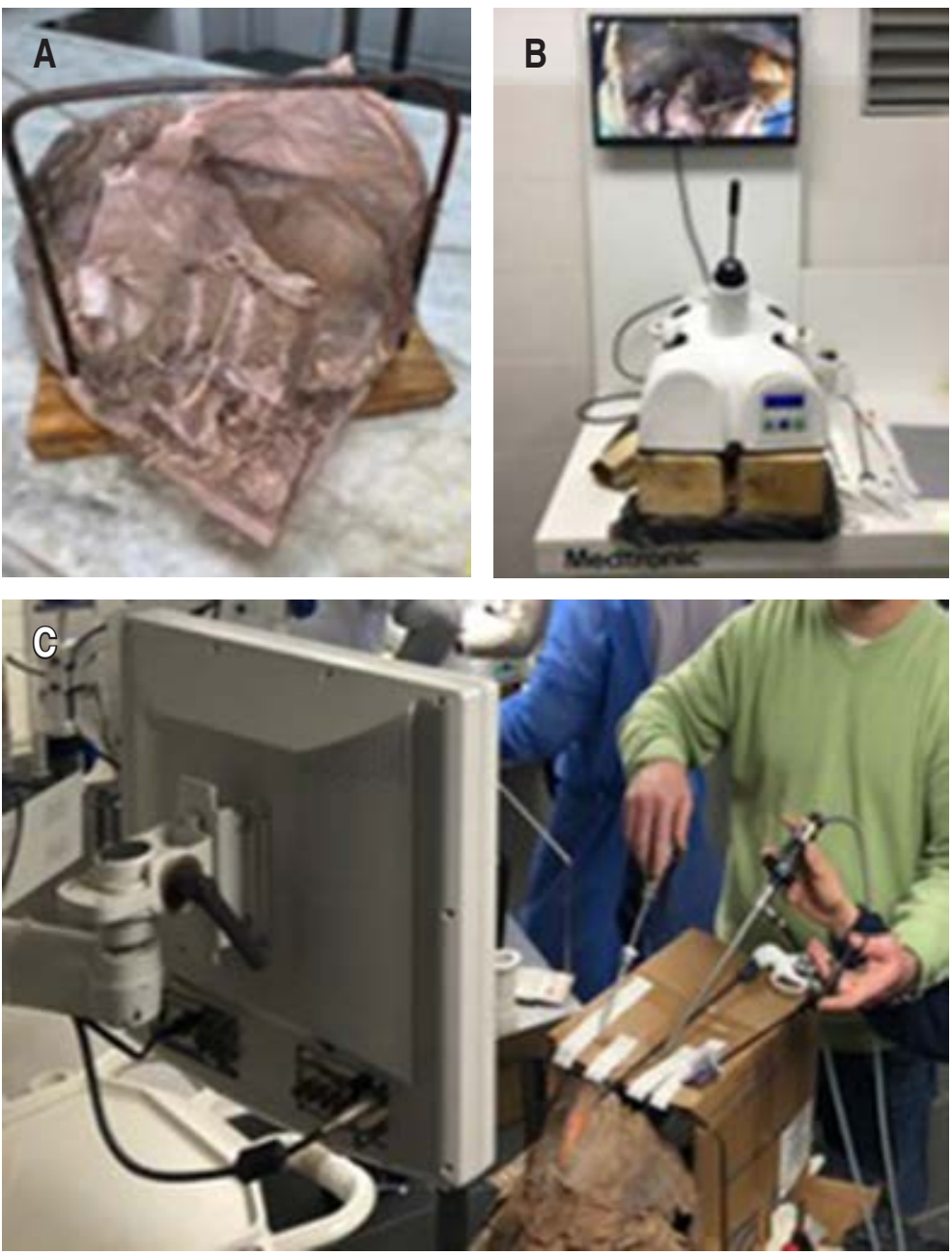

Figura 1: A) Hemipelvis en posición, fija a arco de metal. B) Pelvitrainer con preparado anatómico en su interior. $C$ ) entrenamiento quirúrgico con pelvis entera, fija a arco de metal y con caja de cartón para emplazamiento de trócares.

e instrumental quirúrgico de uso habitual en cirugía laparoscópica y laparotómica.

Para el abordaje laparoscópico, técnica transabdominal preperitoneal (TAAP), utilizamos tanto pelvis enteras como hemipelvis parcialmente evisceradas (se conservaron los órganos del mediastino pélvico, pero se retiraron hacia craneal las asas delgadas y el epiplón mayor). Cada pieza anatómica se colocó imitando la posición operatoria, fijando la pared anterior del abdomen a un arco de metal, para mantenerla en la posición correcta (Figura 1). Tanto utilizando el equipo de laparoscopia con óptica de $30^{\circ}$, como los pelvitrainers (con óptica de cero grados) se realizó paso a paso la técnica quirúrgica, reconociendo las diferentes estructuras anatómicas, hasta exponer el orificio profundo del conducto inguinal, el ligamento de Cooper, etcétera. Se colocó malla de polipropileno, utilizando endotackers para su fijación y poliglactina 910 calibre 3.0 para el cierre del peritoneo (Figura 2).

Como se aprecia en la Figura 1, para poder desarrollar la práctica quirúrgica en los pelvitrainers, fue necesario levantarlos unos $15 \mathrm{~cm}$ (utilizamos bloques de madera), para utilizar el laparoscopio debimos acoplar al arco de metal una caja de cartón como continuación de la pared abdominal, siendo allí donde emplazamos los trócares.

Para el abordaje laparotómico se realizó primero el abordaje de la región inguinoabdominal (incisión oblicua paralela a la arcada crural) y luego el de la región inguinocrural (incisión vertical emplazada $2 \mathrm{~cm}$ por dentro del punto medio de la arcada crural y por debajo de ella). En ambos abordajes se realizó la disección quirúrgica paso a paso, siguiendo estrictamente la técnica quirúrgica, reconociendo las diferentes estructuras anatómicas hasta alcanzar el conducto inguinal y el anillo crural respectivamente.

En la región inguinoabdominal, se realizó primero la hernioplastia de Lichtenstein, utilizando malla de polipropileno, hilo de igual material calibre 2.0 para su fijación, poliglactina 910 calibre 1.0 para el cierre de la aponeurosis del oblicuo mayor y 3.0 para el cierre del celular subcutáneo, y mononylon 4.0 para el cierre cutáneo (Figura 3).

Luego de retirada la malla de polipropileno, se realizó la herniorrafia de Bassini y, luego de deshacer la referida rafia, se realizó la hernioplastia de Shouldice. Utilizando, en ambos procedimientos, polipropileno calibre 2.0 para la rafia parietal (Figura 4).

En la región inguinocrural se realizó primero la hernioplastia de Lichtenstein utilizando plug de malla de polipropileno e hilo de igual material, calibre 2.0, para su fijación, poliglactina 910 calibre 3.0 para el cierre del celular subcutáneo y mononylon 4.0 para el cierre cutáneo. Luego de retirado el plug de malla de polipropileno, se realizó la herniorrafia de Ellis y la de Debin, utilizando polipropileno calibre 2.0 para la rafia parietal.

El equipo quirúrgico, para cada una de las prácticas, estuvo integrado por un instructor y dos residentes por región que iban alternándose en la función de cirujano y ayudante. Participaron ocho residentes y cada uno realizó la totalidad de las reparaciones parietales referidas.

\section{RESULTADOS}

Pudimos reproducir en el cadáver la totalidad de las reparaciones parietales propuestas, si- 
guiendo la secuencia hernioplastia-herniorrafia y realizando primero el abordaje laparoscópico y luego el laparotómico.

La rigidez del material cadavérico no fue un impedimento para la realización de las diferentes prácticas quirúrgicas, pero requirió la realización de abordajes quirúrgicos más amplios y de incisiones de descarga en piel y en la aponeurosis del músculo oblicuo mayor.

Cada residente realizó el entrenamiento de todas las reparaciones parietales referidas, repitiéndolas hasta su sistematización, siendo el instructor quien determinaba cuándo se alcanzaba ese nivel.

Los residentes mostraron interés y estuvieron comprometidos con la realización de este curso de entrenamiento quirúrgico.

\section{DISCUSIÓN}

La secuencia referida, primero plastias y luego rafias, y la realización primero del abordaje laparoscópico y luego el laparotómico nos permitió maximizar la utilización del material cadavérico.

Pudimos realizar, en 22 de 24 regiones inguinales, la totalidad de los procedimientos quirúrgicos mencionados (un TAPP, una plastia y dos rafias inguinoabdominales, una plastia y dos rafias inguinocrurales) o sea que pudimos realizar siete técnicas quirúrgicas por cada hemipelvis, excepto en dos modelos que presentaban adherencias peritoneales, de cirugías previas, que impidieron la realización del ejercicio de hernioplastia TAPP. El entrenamiento del abordaje
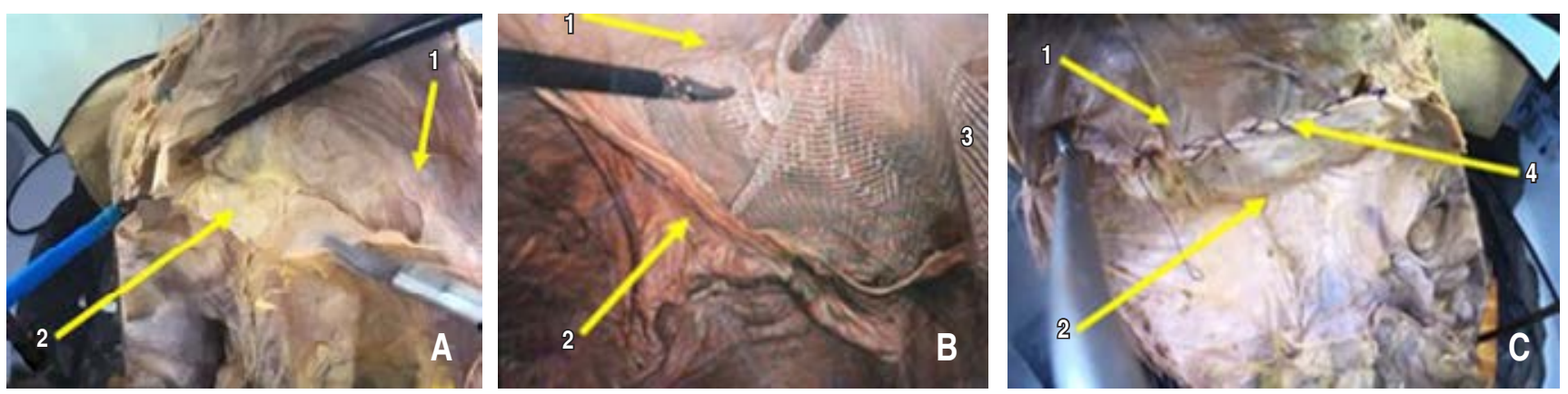

Figura 2: Secuencia de hernioplastia laparoscópica TAAP. A) Disección del peritoneo (flap superior e inferior). B) Colocación de malla de polipropileno. C) Cierre de peritoneo. 1. Flap superior de peritoneo. 2. Flap inferior de peritoneo. 3. Malla de polipropileno. 4. Surjet cerrando ambos flaps de peritoneo.
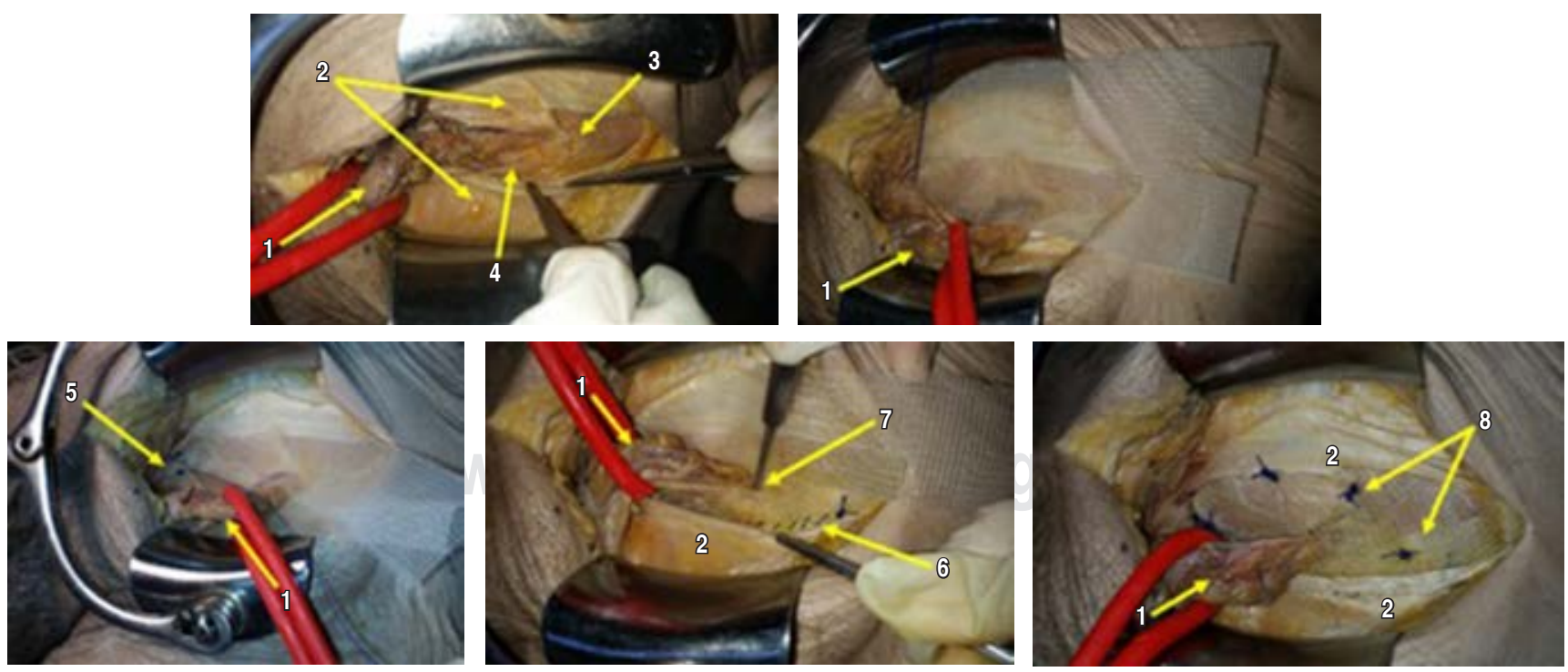

Figura 3: Secuencia de hernioplastia de Lichtenstein. 1. Cordón espermático. 2. Aponeurosis del oblicuo mayor. 3. Orificio inguinal profundo. 4. Nervio abdominogenital mayor. 5. Punto en la espina del pubis. 6. Surjet en la cintilla iliopubiana. 7. Entrecruzamiento de flaps. 8. Resto de los puntos para fijación de la malla. 

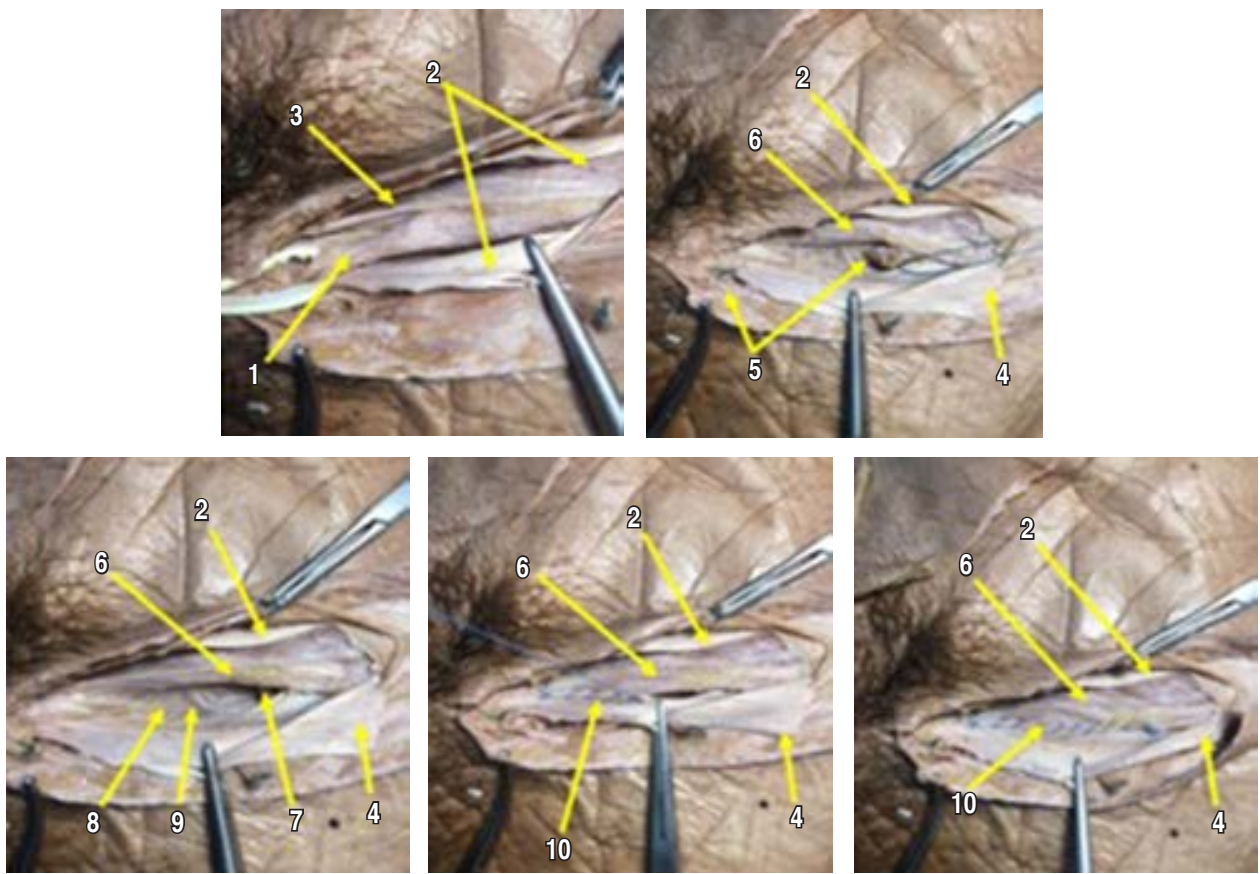

Figura 4:

Secuencia de herniorrafia de Bassini. 1. Ligamento redondo. 2. Aponeurosis del oblicuo mayor. 3. Nervio abdominogenital mayor. 4. Incisión de descarga en aponeurosis del oblicuo mayor. 5. Muñón del ligamento redondo (ya resecado). 6. Tendón conjunto. 7. Orificio inguinal profundo. 8. Fascia transversalis. 9. Vasos epigástricos. 10. Descenso del tendón conjunto a la arcada crural. quirúrgico laparotómico pudo realizarse en las 24 regiones utilizadas.

La realización de abordajes más amplios y/o de incisiones de descarga nos permitió desarroIlar el ejercicio de entrenamiento y evitar que la rigidez del material constituyera una limitante, estando a cargo del instructor el señalar que estas maniobras no forman parte de la técnica quirúrgica y que se realizan sólo por necesidad. Lo ideal sería contar con material cadavérico flácido, sea cadáver fresco o fijado (por ejemplo, con técnica de Thiel), pero por el momento no disponemos del mismo.

El instructor, además de ir señalando los puntos clave de cada paso quirúrgico, fue evaluando las destrezas del residente en el uso de instrumental quirúrgico, manejo de hilos de sutura, manipulación de tejidos y de la malla, así como el conocimiento de cada técnica quirúrgica. Durante cada ejercicio, el instructor fue señalando y corrigiendo errores, realizando una síntesis de todo lo señalado al finalizar cada ejercicio. Al repetir el ejercicio, se prestó especial atención a que no ocurrieran nuevamente los mismos errores. No se realizó una evaluación cuantitativa de cada uno de los puntos mencionados.

Los residentes pudieron familiarizarse con la anatomía quirúrgica de la región inguinal tanto en su abordaje laparotómico como laparoscópico. Pudieron reproducir paso a paso cada una de las técnicas quirúrgicas. Realizaron y sistematizaron cada técnica quirúrgica en iguales condiciones espaciales y físicas que en el paciente.

\section{CONCLUSIONES}

El cadáver humano es un excelente fantoma para el entrenamiento quirúrgico. Constituye un modelo de alta calidad y fidelidad para el entrenamiento en herniorrafias y hernioplastias inguinales por abordaje laparotómico o laparoscópico.

La rigidez del material formolado, si bien no constituye una limitante para el ejercicio de simulación, requiere la realización de abordajes más amplios y de incisiones de descarga.

\section{REFERENCIAS}

1. Jakimowicz JJ, Jakimowicz CM. Simulación en cirugía, ¿dónde estamos y a dónde llegaremos? Cir Cir. 2011; 79: 44-49.

2. Rosen RK. The history of medical simulation. J Crit Care. 2008; 23: 157-166.

3. Aggarwal R, Darzi A. Technical-skills training in the $21^{\text {st }}$ century. N Engl J Med 2006; 355 (25): 2664-2669.

4. León F, Varas J, Buckel E, Crivani F, Pimentel F, Martínez, F, et al. Simulación en cirugía laparoscópica. Cir Esp. 2015; 93 (1): 4-11.

5. Yhomas M. The role of simulation in the development of technical competence during surgical training: a literature review. Int J Med Educ. 2013; 4: 48-58. 
6. Ruíz J, Martín J, González M, Redondo C, Manuel J. La simulación como modelo de enseñanza en cirugía. Cir Esp 2018; 96 (1): 12-17.

7. Sutherland L, Middleton P, Anthony A, Hamdorf J, Cregan P, Scott D, et al. Surgical simulation: a systematic review. Ann Surg. 2006; 243: 291-330.

8. Selcuk I, Tatar I, Huri E. Cadaveric anatomy and dissection in surgical training. Turk J Obstet Gynecol. 2019; 16: 72-75.

9. Yiasemidon M, Gkaragkani E, Glassman D, Biyani C. Cadaveric simulation: a review of reviews. Ir J Med Sci. 2018; 187: 827-833.

10. Rodríguez R, Losardo R, Binvignat O. LA anatomía humana como disciplina indispensable en la seguridad de los pacientes. Int J Morphol. 2019; 37 (1): 241-250.

11. Pélissier $E$. Anatomie chirurgicale des hernies de l'aine. Encycl Méd Chir. Techniques chirurgicales-Appareil digestif. 2000; 40-105.
12. Dulucq J, Himpens J. Traitement des hernies inguinales par laparoscopie: chirugie laparoscopique totalement extrapéritonéale; chirugie laparoscopique transabdominale prépéritonéale. Techniques chirurgicales-Appareil digestif. EM Consulte. 1996; 40-137.

13. Pélissier E, Marre P, Damas J. Traitement des hernies inguinales. Choix d'un procédé. Techniques chirurgicalesAppareil digestif. EM Consulte. 2000; 40-138.

14. Pélissier E, Marre P. Traitement chirurgicale des hernies crurales. Choix d'un procédé. Techniques chirurgicalesAppareil digestif. EM Consulte. 2000; 40-107.

Correspondencia:

Dr. Gustavo Armand Ugon-Bigi

Agustina Contucci de Oribe 2498,

Montevideo, Uruguay, 00598-99244795.

E-mail: gusad@adinet.com.uy 